Horizons philosophiques

\title{
Cinéma et diversité culturelle : le cinéma indépendant face à la mondialisation des industries culturelles
}

\section{Damien Rousselière}

Volume 15, numéro 2, printemps 2005

Cultures ou mondialisation?

URI : https://id.erudit.org/iderudit/801295ar

DOI : https://doi.org/10.7202/801295ar

Aller au sommaire du numéro

Éditeur(s)

Collège Édouard-Montpetit

ISSN

1181-9227 (imprimé)

1920-2954 (numérique)

Découvrir la revue

Citer cet article

Rousselière, D. (2005). Cinéma et diversité culturelle : le cinéma indépendant face à la mondialisation des industries culturelles. Horizons philosophiques, 15(2), 101-123. https://doi.org/10.7202/801295ar d'utilisation que vous pouvez consulter en ligne. 


\section{Cinéma et diversité culturelle : le cinéma indépendant face à la mondialisation des industries culturelles}

Actuellement, le panorama du cinéma comme art et «par ailleurs industrie" (Malraux, 1946) se transforme fortement, exacerbant les contraintes économiques dans lesquelles prennent place les œuvres cinématographiques. Parallèlement aux problèmes souvent mis en évidence de la nouvelle économie de la production du cinéma ${ }^{1}$, cette évolution se traduit par une réorganisation de la filière de distribution et de diffusion, et notamment la concentration des diffuseurs. Ainsi l'émergence forte des multiplex, représentant $25 \%$ des écrans en Europe (OEA, 2003) ${ }^{2}$, conduit au fait qu'en France dorénavant $4 \%$ des établissements comptent pour environ $40 \%$ des entrées. En réaction à ces stratégies des circuits nationaux mais aussi régionaux, des regroupements de salles indépendantes d'art et d'essai - qui représentent $25 \%$ de la fréquentation - se mettent en place face à ces nouvelles exigences de la diffusion et distribution [(Demoustier, Rousselière, Cassier, Clerc, 2003) et (Rousselière, 2004 a et b)]. Comme le remarquent différents auteurs, les industries culturelles conduisent à la fois à l'inflation du nombre d'œuvres produites et à la réduction concomitante de leur diffusion et exposition au public, posant d'une manière nouvelle la question de la défense de la diversité culturelle et des politiques publiques visant cet objectif. En effet à bien des égards ce sont les structures de diffusion et de distribution qui, en connaissant les mêmes phénomènes de convergence, concentration et massification que les structures de production, favorisent le plus l'homogénéisation culturelle (Benghozi, 2003) ${ }^{3}$. À ce titre, cette réorganisation de la filière du cinéma pose de manière accrue la question de la pérennité d'un circuit indépendant de diffusion (à la fois comme cinéma de proximité et structures d'éducation à l'image) et du rôle important qu'il puisse jouer dans la préservation d'une diversité culturelle cinématographique. Ce dernier objectif, explicitement revendiqué au niveau français, européen ou mondial, est la nouvelle norme à laquelle doivent correspondre les politiques publiques (Mattelart, 2002). 
Nous nous inscrivons ici dans une approche en économie politique malgré le risque de réductionnisme qui lui est systématiquement accolé [(Créton, 1998, 2001), (Mattelart, Neveu, 1996, 2003), (Wayne, 2003)]. Ainsi cette approche dès lors qu'on ne la réduit pas à "l'économisme" (i.e. un matérialisme "vulgaire» conduisant à ce que tout (superstructures) soit expliqué par l'analyse matérielle des conditions de production (infrastructures) ${ }^{4}$ ) permet de donner sens à la double nature du cinéma 5 . On est en effet renvoyé à la question de l'économie spécifique des biens symboliques (Bourdieu, 1994) : la dynamique de la relation art-industrie explique les caractéristiques spécifiques de la gestion dans le secteur cinématographique. En tant qu'art moderne par excellence, car mobilisant une chaîne industrielle de production, la dialectique coopération-antagonisme y est nécessairement à l'œuvre et partant les variables techniques, artistiques, organisationnelles, managériales doivent être combinées et s'inscrire dans des processus de régulation, d'arbitrage et de décision. Ce débat propre aux industries culturelles s'explique par des compromis entre plusieurs registres de justification, du fait de la cohabitation de plusieurs "cités" (Boltanski, Thévenot, 2000). La légitimité économique de l'entreprise, qui s'impose d'elle-même par les impératifs commerciaux, financiers et de compétitivité, est confrontée à une autre légitimité symbolique et artistique dont les fondements ne sont pas du même ordre (dimension industrielle/ marchande versus dimension artistique ou de l'inspiration).

Dans ce cadre, cet article a pour objet d'établir un diagnostic de la nouvelle place et du rôle potentiel du circuit de diffusion indépendant. Nous abordons donc ce thème en deux grandes étapes. Dans un premier temps il s'agit de voir dans quelle mesure la transformation actuelle de l'activité d'exploitation cinématographique (s')accompagne d'une transformation de la nature des films proposés, questionnant alors la notion de diversité culturelle non seulement au plan quantitatif (part des films suivant leur nationalité) mais aussi au plan qualitatif (prédominance d'un rapport particulier à l'image) ${ }^{6}$. Dans un second temps, nous nous intéresserons au problème alors de la situation spécifique du cinéma indépendant et de sa composante diffusion. En conclusion nous nous interrogerons à partir de ce diagnostic sur le renouveau possible des politiques publiques. 


\section{Le cinéma : art et/ou industrie?}

\section{La transformation de la filière industrielle}

La situation européenne montre la prééminence d'une forme de cinéma, approchant au plan quantitatif cette notion de diversité culturelle.

\section{Tableau 1 : Parts de marché des films distribués dans I'Union européenne (1996-2002)}

\begin{tabular}{|l|c|c|c|c|c|c|c|}
\hline \multirow{2}{*}{ Films US } & \multicolumn{1}{|c}{1996} & 1997 & 1998 & \multicolumn{1}{c}{1999} & 2000 & 2001 & 2002 \\
\cline { 2 - 8 } & $71,6 \%$ & $67,0 \%$ & $77,5 \%$ & $69,2 \%$ & $73,3 \%$ & $65,4 \%$ & $71,2 \%$ \\
\hline $\begin{array}{l}\text { Films } \\
\text { nationaux sur leur propre marché }\end{array}$ & $17,1 \%$ & $21,3 \%$ & $14,4 \%$ & $17,5 \%$ & $15,7 \%$ & $20,1 \%$ & $19,5 \%$ \\
\hline $\begin{array}{l}\text { Films } \\
\text { européens hors marché national }\end{array}$ & $8,8 \%$ & $10,7 \%$ & $7,1 \%$ & $11,3 \%$ & $7,2 \%$ & $11,0 \%$ & $7,9 \%$ \\
\hline Autres & $2,6 \%$ & $1,1 \%$ & $1,1 \%$ & $2,0 \%$ & $3,8 \%$ & $3,5 \%$ & $1,3 \%$ \\
\hline
\end{tabular}

Source : Observatoire européen de l'audiovisuel (http://lumiere.obs.coe.int)

La base de données Lumière de l'Observatoire européen de l'audiovisue/permet de mettre en évidence que si les titres américains ne représentent que $39 \%$ à $46 \%$ de l'offre de films, ils représentent selon les pays entre 56 et $83 \%$ des entrées, et ceci alors même que les films produits dans l'Union européenne représentant la moitié de l'offre ne réalisent que de un quart à un tiers des entrées et que les films du reste du monde entre 1 et $4 \%$ selon les années. II semble donc bien que la question de la diversité culturelle ne se pose donc pas seulement dans la production mais bien dans la réception du spectacle cinématographique et dans l'égal accès de tous les types de films à une certaine exposition.

Le panorama de l'exploitation en France et en Europe met en évidence une structure de marché oligopolistique, marquée par la présence de groupements de programmation nationaux, modifiée récemment par le regroupement des activités d'exploitation de salles de Gaumont et de Pathé sous l'enseigne EuroPalaces en 2001 pour désormais $13,1 \%$ des écrans français. L'ensemble des groupements nationaux programme près de $34 \%$ des écrans actifs. Dans le cadre de cette tendance à une concertation forte des réseaux de diffusion, l'évolution de la répartition de la recette sur la longue période est un 
bon indice des rapports de force au sein de l'industrie cinématographique.

Tableau 2 : Évolution de la répartition de la recette (1964-2000)

\begin{tabular}{|l|c|c|c|c|c|c|c|c|c|c|c|}
\hline & 1964 & 1971 & 1975 & 1980 & 1985 & 1990 & 1994 & 2000 & $\begin{array}{l}\text { Évolution } \\
\text { Évolution }\end{array}$ \\
\cline { 2 - 12 } & 22,2 & 14,5 & 14,3 & 7 & 6,7 & 5,3 & 5,3 & 5,3 & $-76,13 \%$ & $0,00 \%$ \\
\hline Fisc & Exploitation & 45,9 & 50 & 49,2 & 50,7 & 48,1 & 43,1 & 42,6 & 41,05 & $-10,57 \%$ & $-4,76 \%$ \\
\hline $\begin{array}{l}\text { Production/ } \\
\text { Distribution }\end{array}$ & 29,2 & 33,5 & 35 & 40,9 & 43,4 & 39,2 & 40 & 41,47 & $42,02 \%$ & $5,79 \%$ \\
\hline SACEM & 1,4 & 2 & 1,6 & 1,5 & 1,4 & 1,3 & 1,3 & 1,27 & $-9,29 \%$ & $-2,31 \%$ \\
\hline Taxe spéciale & & & & & & 11,1 & 11,8 & 10,91 & & $-1,71 \%$ \\
\hline
\end{tabular}

Sources : (Bonnell, 2001) et CNC

Le tableau précédent montre ainsi que si l'exploitation a longtemps perçu la proportion la plus importante de la recette, sa situation va progressivement se dégrader ${ }^{7}$. Partant d'une position assez basse (30\% en moyenne dans les années soixante), la part de la production et de la distribution va lentement améliorer sa position vis-à-vis de la salle pour atteindre la parité avec elle ${ }^{8}$. Phénomène traditionnellement analysé en économie industrielle, le processus de concentration à l'aval conduit à une transformation des rapports de force en faveur d'une part de ces grandes sociétés de distribution et d'autre part des grandes sociétés d'exploitation, seules à même de négocier dans de bonnes conditions avec les précédentes. Tendent à se constituer ainsi deux grands pôles dans le cinéma : le processus de concentration de l'exploitation, secteur encore relativement éclaté, étant conforté par la concentration de la distribution, comme avec les alliances de distribution entre Gaumont et Buena Vista international, et entre Fox et Pathé. 


\section{Tableau 3 : Situation de la concentration de la distribution et de l'exploitation en France en 2003}

Les 5 premiers distributeurs

(en termes d'encaissements/ distributeur)
Les 5 premiers exploitants

(groupements nationaux de programmation)

\begin{tabular}{|c|c|}
\cline { 2 - 2 } \multicolumn{1}{c|}{} & $\begin{array}{c}\text { Part de marché } \\
\text { (en \%) }\end{array}$ \\
\hline GBVI & 15,3 \\
\hline UIP & 11,9 \\
\hline Warner Bros & 11,7 \\
\hline UFD & 8,8 \\
\hline $\begin{array}{c}\text { Metropolitan } \\
\text { Filmexport }\end{array}$ & 7,4 \\
\hline Total & 55,1 \\
\hline
\end{tabular}

Source : CNC

\begin{tabular}{|c|c|c|}
\cline { 2 - 3 } \multicolumn{1}{c|}{} & $\begin{array}{l}\text { Nombre } \\
\text { d'écrans }\end{array}$ & $\%$ du Parc \\
\hline EuroPalaces & 659 & 12,4 \\
\hline UGC & 428 & 8,1 \\
\hline SOREDIC & 229 & 4,3 \\
\hline SAGEC Ciné 32 & 218 & 4,1 \\
\hline GPCI & 124 & 2,3 \\
\hline Total & 1658 & 31,2 \\
\hline
\end{tabular}

Dans ce contexte de fortes mutations de l'exploitation, deux éléments importants de dérégulation et d'exacerbation de la concurrence sont apparus. Le premier est relatif aux cartes illimitées qui depuis le lancement, en 2000 , de "formules d'accès au cinéma donnant droit à des entrées multiples" par les principaux circuits nationaux, a bouleversé tant les relations entre l'amont et l'aval de la filière cinématographique ${ }^{9}$ que les conditions de fréquentation et d'exploitation des salles de cinéma, ces "cartes illimitées" étant davantage accessibles aux grands circuits cinématographiques et aux opérateurs de multiplex qu'aux petits et moyens exploitants. Plus fondamentalement, l'introduction des cartes a entraîné un changement de la consommation du cinéma (sur le modèle des téléphones mobiles), introduisant ce que les spécialistes nomment le marketing viral dans le secteur, et instaurant une relation directe entre les spectateurs et les opérateurs de cartes, à présent capables de cibler au mieux les attentes de leurs clients. La consommation des abonnés est ainsi aujourd'hui de plus en plus déterminée par leur appartenance à un circuit d'exploitation puisque $85 \%$ des abonnés utilisent leur carte ${ }^{10}$. Les cartes d'abonnement s'inscrivent par ailleurs dans une stratégie de dumping, favorisant la concentration des entreprises de distribution, car elles ne sont pas actuellement rentables. C'est pour ces raisons que les pouvoirs publics ont souhaité réguler 
(en partie) ces cartes en instituant une procédure d'agrément, imposant la transparence de la remontée de la recette et l'ouverture aux autres exploitants des formules mises en place par des exploitants économiquement dominants.

Pris isolément ce premier élément n'a pas nécessairement un impact négatif sur la diversité culturelle puisqu'il peut conduire à valoriser des stratégies de niches et de diversification / spécialisation des circuits cinématographiques, proposant alors des «biens clubs» à un ensemble restreint mais fidélisé de clients - sur le modèle des chaînes de télévision par abonnement (Hansmann, 1996). Toutefois il suit un autre mouvement plus fondamental : celui des multiplex ${ }^{11}$, dont la logique a avant tout été portée par les grandes entreprises du secteur. Ainsi en France, c'est le circuit d'exploitation Pathé qui, le premier, s'est engagé dans cette politique. Les deux autres grands circuits nationaux, UGC et Gaumont, ont suivi le mouvement d'investissement, ce qui leur a permis de se prémunir contre le risque d'une baisse de leurs parts de marché qui aurait pu naître de l'arrivée sur le marché français d'opérateurs étrangers. Puis leur stratégie d'investissement est devenue plus offensive au fur et à mesure que les bons résultats obtenus par les multiplex confirmaient la rentabilité de ces équipements. S'il a été entamé plus tardivement en France que dans les autres pays européens, le processus n'a cessé de s'accélérer, jusqu'à s'étendre aujourd'hui à des villes moyennes, les grandes agglomérations étant d'ores et déjà quasiment toutes pourvues en multiplex. Ce phénomène est également porté par les Groupements régionaux du secteur tendant pour défendre leurs parts de marché à adopter le même type de stratégie, en atteste par exemple le cas du groupe grenoblois Adira d'origine familiale, qui développe cette stratégie à l'échelle régionale. 


\section{Graphique 1 : Part des multiplex dans l'exploitation cinématographique française (1996-2003)}

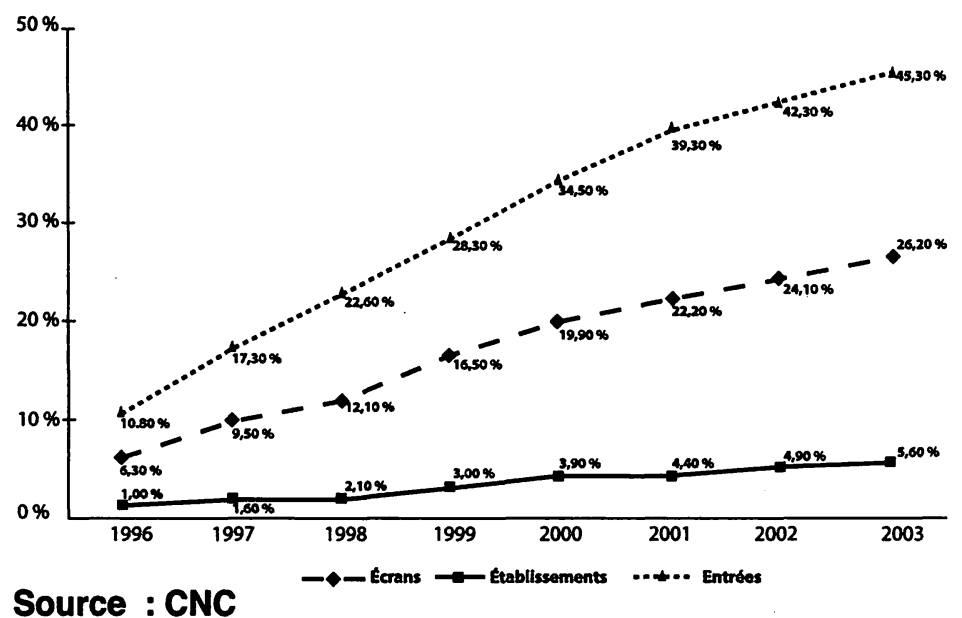

Les stratégies de création de multiplex sont toutefois différentes suivant les pays :

"en Grande-Bretagne, le développement des multiplex, entamé à partir de 1985, a principalement été le fait des majors américaines. En revanche, l'emprise des grands groupements nationaux sur l'exposition, le poids économique du secteur, la forte politisation du cinéma, et sa centralité à l'identité culturelle nationale dans l'imaginaire collectif ont constitué d'importants obstacles à la capacité de Warner, National Amusements, American Multi-Cinemas ou UCl à poser leur enseigne symbolique sur le territoire français" (Hayes, 2003 : 4).

Ainsi bien qu'ils n'en soient pas les seuls acteurs, les groupements nationaux de programmation sont les principaux promoteurs de multiplex en France. La principale raison réside dans le coût élevé des investissements nécessaires à la construction d'un multiplex (entre 10 et 15 millions d'euros) ainsi que dans les frais liés à son fonctionnement (entre 20 et 40 salariés), éléments que seul un grand groupe du fait de son réseau peut se permettre. En effet selon Claude Forest (2002), les trois-quarts des multiplex pris isolément n'atteignent pas le seuil de rentabilité, la faiblesse des cofinancements publics n'autorisant pas en outre ce type de stratégie pour un 
acteur indépendant. L'implantation des multiplex s'inscrivant dans une stratégie explicite par les coûts et les volumes correspond bien à une logique de consolidation des trois principales sociétés françaises (en atteste le nombre croissant de salles dont ils sont propriétaires).

Dans ce cadre général, on assiste à de nouvelles stratégies de concentration et d'intégration de la filière et surtout un encombrement des films pour l'accès aux salles. Comme l'a montré Claude Forest (2002 : 149), du fait de l'accélération de l'usure économique du film, et compte tenu du coût marginal d'une copie (amortie en réalisant seulement 500 à 700 entrées), les distributeurs ont augmenté le nombre de copies disponibles, faisant ainsi passer l'ensemble du marché de la rareté à l'abondance. Ainsi plus de la moitié des nouvelles sorties se font sur plus de 500 salles raréfiant d'autant la possibilité pour d'autres films d'être programmés dans de bonnes conditions ${ }^{12}$. La programmation dite «au lundi»13 conduit à ce que l'étalonnage de la réussite des films va progressivement s'établir en fonction des entrées réalisées sur ses cinq premiers jours d'exploitation. Cela s'opère au détriment des salles les plus faibles, mais surtout des films qui affichent des résultats inférieurs en valeur relative à ceux de leurs concurrents du moment, accélérant leur disparition du marché. Sur le type de cinéma valorisé, les multiplex renforcent «l'effet podium» sur la carrière des films, en jouant un rôle d'amplificateur de succès ou d'échec; ce n'est donc alors pas l'exposition au public des films (l'accès aux salles) qui risque d'être compromise, mais leur durée de vie et donc leur rendement total ${ }^{14}$.

Enfin, les multiplex développent un nouveau cadre de "consommation" du cinéma : selon une étude du CNC portant sur les publics des multiplex les arguments majeurs qui jouent en faveur des multiplexes portent essentiellement sur la qualité des salles, de l'écran, du son et le confort des fauteuils et les facilités d'accès, les possibilités de stationnement (rappelons que $81 \%$ des spectateurs viennent avec leur véhicule personnel), alors même qu'ils sont souvent plus chers que les autres cinémas. Comme le remarque justement G. Hayes (2003), les multiplex transforment les cinémas en centres de divertissement familial, fournissant des possibilités de consommation sans l'obligation d'interaction, l'imprévisibilité et la diversité culturelle de la cité : à la place du film-plus-débat, le multiplex offre la formule «sortie» (voiture-fast-food-cinéma ${ }^{15}$ ) dans un endroit intégré et sécurisé, à proximité de grands centres commerciaux, à l'intérieur des salles les spots publicitaires ayant déjà 
remplacé en grande partie les bandes annonces. S'appuyant sur une division du travail inspirée de la restauration rapide (avec ses procédés de déqualification et de parcellisation du travail), ils en transposent un mode de consommation auquel sont désormais acculturés les «fils de Mc Do» (Ariès, 1997).

\section{... à une nouvelle esthétique du cinéma?}

De manière générale, cette massification de la production et la concentration des entreprises d'exploitation conduisant à la réduction des chances d'exposition s'inscrit plus généralement dans l'économie du star-system [(Benhamou 2003) et (Rosen 1981)], ayant pour effet, quelle que soit l'industrie culturelle considérée, d'aboutir à une concentration de la consommation culturelle sur un nombre d'œuvres de plus en plus restreint. Dans un tel contexte, la diversité culturelle peut sembler effectivement préservée du fait du volume très important des œuvres produites et du flux continuel de nouveautés, alors qu'en réalité elle est mise en question par la réduction du nombre des œuvres effectivement mises à la disposition du public. Ce poids de la distribution et de la massification de la culture sur les contenus culturels est d'autant plus fort que les biens culturels relèvent d'une économie des biens immatériels conduisant à de fortes économies d'échelle (coûts de reproduction pratiquement nuls, même si des coûts de commercialisation peuvent alors être importants) et à des effets de réseaux ou d'économies d'envergure du fait de la complémentarité entre certains biens (spectacle vivant et industries culturelles par exemple, ou au sein des industries culturelles) ${ }^{16}$.

Dans ce cadre, comme le montre le tableau récapitulatif ci-après, les États-Unis bénéficient d'un large marché intérieur (unifié, contrairement à l'Europe) permettant d'amortir les coûts de leur production et d'être offensifs sur les marchés d'exportation, aussi bien au plan des tarifs (souvent inférieurs à celui des productions nationales) que des actions de promotion (l'amortissement préalable leur permet en effet de dégager des ressources auxquelles les producteurs des autres pays n'ont pas accès) : «aux États-Unis, un marché intérieur, abrité de fait et associé à une production quantitativement maîtrisée, permet aux producteurs de financer des films à plus gros budgets, inaccessibles aux producteurs étrangers et qui confortent la situation de quasi-monopole des États-Unis sur le marché des superproductions" (Siroën, 2000 : 107). Ces caractéristiques économiques engagent alors le cinéma dans une spirale 
infernale (Benghozi, 2003) : les économies d'échelle et la massification de la distribution incitent au développement d'actions promotionnelles et commerciales qui favorisent les producteurs disposant de moyens importants et les produits visant les marchés les plus larges, ce qui renforce encore la banalisation et la standardisation des produits, même quand ils sont ancrés dans un terreau culturel spécifique, mettant en évidence la capacité souvent remarquée de "Hollywood" à recycler les cultures locales : la liste des cinéastes publiée par les Cahiers du Cinéma en 2003 (des auteurs de Hong-Kong comme Tsui Hark ou John Woo aux cinéastes français comme Jeunet ou Kassovitz) opérant avec des moyens américains met ce phénomène en évidence ${ }^{17}$.

Par rapport aux autres industries culturelles, le cinéma présente une situation où à la concentration capitalistique s'ajoute une concentration géographique, puisque les États-Unis investissent plus dans le cinéma que l'ensemble des autres pays et que pour les cent pays ayant aujourd'hui une activité de production cinématographique, 30 produisent plus de 20 films par an et 5 seulement plus de 200. Si la production est concentrée géographiquement, et ceci même à l'intérieur des pays (Scott, 2000), l'hégémonie culturelle que l'on attribue à "Hollywood" s'accompagne en outre parfaitement d'une diversité des capitaux des entreprises (Reich, 1992) puisque au "cours des deux dernières décennies, toutes les majors étatsuniennes ont fait l'objet de rachats et d'intégration au sein de groupes de communication à vocation mondiale" (Forest, 2002 :212). 
Tableau 4 : Panorama mondial du cinéma en 2002

\begin{tabular}{|c|c|c|c|c|c|c|c|}
\hline & Allemagne & Espagne & France & Italie & $\begin{array}{c}\text { Royaume } \\
\text { Uni }\end{array}$ & Etats Unis & Japon \\
\hline $\begin{array}{l}\text { Population totale } \\
\text { (millions) }\end{array}$ & 82,4 & 40,4 & 59,5 & 57,8 & 60,1 & 288,4 & 127,5 \\
\hline $\begin{array}{l}\text { Nombre de foyers } \\
\text { (millions) }\end{array}$ & 39,6 & 13,7 & 24,7 & 22,5 & 26,2 & 112,1 & 50,6 \\
\hline $\begin{array}{l}\text { Longs métrages } \\
\text { (y compris les films di }\end{array}$ & \begin{tabular}{|c|}
84 \\
soproductiol
\end{tabular} & 114 & 200 & 130 & 119 & 543 & 293 \\
\hline $\begin{array}{l}\text { Nombre de longs } \\
\text { métrages par million c }\end{array}$ & $\begin{array}{r}1,02 \\
\text { labitant }\end{array}$ & 2,82 & 3,36 & 2,25 & 1,98 & 1,88 & 2,3 \\
\hline Écrans & 4868 & 4039 & 5265 & 3299 & 3248 & 35280 & 2635 \\
\hline Entrées (millions) & 163,9 & 140,7 & 184,2 & 111,5 & 175,9 & 1639,3 & 160,8 \\
\hline Indice de fréquentation. & 2 & 3,5 & 3,1 & 1,9 & 2,9 & 5,7 & 1,3 \\
\hline $\begin{array}{l}\text { Recettes guichet } \\
\text { (millions d'euros) }\end{array}$ & 960,1 & 625,9 & 1027,7 & 629,4 & 1187,2 & 7841,5 & 1488,1 \\
\hline $\begin{array}{l}\text { Prix moyen du ticket } \\
\text { en euros }\end{array}$ & 5,86 & 4,45 & 5,58 & 5,64 & 6,75 & 4,78 & 9,25 \\
\hline $\begin{array}{l}\text { Dépense moyenne } \\
\text { par habitant en euros }\end{array}$ & 11,72 & 15,57 & 17,30 & 10,73 & 19,57 & 27,27 & 12,03 \\
\hline $\begin{array}{l}\text { Part du film national } \\
\text { (\% des recettes) }\end{array}$ & 9,5 & 13,7 & 34,8 & 22,2 & 8,3 & 95,6 & 27 \\
\hline $\begin{array}{l}\text { Part du film américain } \\
\text { (\% des recettes) }\end{array}$ & 77,3 & 66,1 & 50,1 & 60,2 & 71,3 & 95,6 & 69,4 \\
\hline
\end{tabular}

Source : CNC

Ce dernier élément, l'intégration du cinéma dans une économie plus large de l'audiovisuel, conduit à renforcer la part de plus en plus importante des télévisions et de l'esthétique qu'elles imposent. Ainsi est enclenché un cercle vicieux : "quand le financement du cinéma dépend à ce point des chaînes de télévision, ces dernières arrivent à imposer leur esthétique... qui, bien souvent, se résume au casting ${ }^{18}$ Le dépassement du "dessus de ligne" provoque un effet boule de neige sur tous les postes et entraîne les films dans une autre économie" (Prado, 2003 : 27). Toutefois de même les entreprises cinématographiques sont fortement diversifiées, quelques rares exceptions de télévisions indépendantes, Arte en Allemagne et en France, $B B C$ en Angleterre ou VPRO aux Pays-Bas contribuent à la défense d'une certaine diversité culturelle ${ }^{19}$. Pour reprendre l'analyse en termes de champs de Pierre Bourdieu (1996), on voit ici alors que le processus d'intégration de cette économie de l'audiovisuel se fait par constitution d'un pôle regroupant les agents dominants du champ du cinéma (les majors, les grands studios) et ceux de l'audiovisuel (les grands chaînes privées telles TF1 ou Canal +), laissant une place 
à un pôle dominé (chaînes (publiques) culturelles et producteurs indépendants). Mais de même que les agents du champ littéraire forment système en tissant des rapports ambivalents entre eux (Bourdieu, 1998), on peut alors se demander dans quelle mesure la préservation de ce pôle indépendant dominé économiquement (constitué en ce qui concerne les télévisions essentiellement d'acteurs publics - sauf pour VPRO) même fortement doté en capital culturel ne sert-il pas de justification aux stratégies hégémoniques des agents dominants du champ audiovisuel et cinématographique?20

Dans le cadre d'un secteur du cinéma en salle en déclin mais dans celui en explosion des secteurs dérivés de la consommation à domicile21, cette nouvelle économie de l'audiovisuel permet de donner pleine réalité aux stratégies multimédia du merchandising des entreprises de loisirs prenant acte de la diversification des sources de profits; la concurrence ne se faisant plus que sur un support multimédia particulier mais sur un ensemble (cinéma, DVD, Télévision, Jeux Vidéo). L'exemple idéal-typique étant Matrix dont l'œuvre est composé du film, de la série d'animation et du jeu vidéo, qui n'en sont plus de simples déclinaisons 22 . Ce type de stratégie multimédia favorise ainsi un type de cinéma, qui pour reprendre la division de Claude Chabrol (2003) entre "conteurs et poètes", est avant tout tourné vers le récit et sa lisibilité, l'histoire à raconter, vu comme un facteur clé du succès des films ${ }^{23}$. En témoigne, comme le note Joëlle Farchy (1999), la multiplication des remakes de succès anciens, des suites, des adaptations de séries, des films "d'acteurs" ou réunissant des acteurs connus..., c'est-à-dire des films s'inscrivant dans une stratégie de rreproculture" car basés sur un univers existant a priori et donc plus facilement reconnaissables pour le consommateur (pouvant toutefois laisser encore une certaine marge de manœuvre / autonomie à l'auteur (Jousse, 2003 : 221-222)24. Cela permet d'une part d'éliminer l'incertitude - dans l'esprit du futur spectateur - sur la qualité supposée du produit (Benhamou, 2003) et d'autre part de favoriser les politiques commerciales associées. Si on suit les analyses de G. Hayes (2003) et M. Wayne (2003), l'impact global de la nouvelle économie du cinéma est double : sur le type de cinéma valorisé par cet intermédiaire, qui comme forme particulière de "mythe et de rêve" tend à l'imposition d'un certain "homme imaginaire" (Morin, 1956), et sur le rapport au cinéma qui est valorisé, entrant dans le cadre d'une "macdonalisation du monde» (Ariès, 1997). 


\section{Quel cinéma indépendant dans la mondialisation?}

La transformation de l'économie «dominante» du cinéma tend à interroger le rôle et la fonction des circuits indépendants d'exploitation, largement constitué en France par le réseau des salles d'art et d'essai. En 2003, celles-ci sont au nombre de 873 établissements de cinéma représentant $25 \%$ de la fréquentation. Ce classement «art et essai» se fait par établissement et prend en compte la proportion de séances réalisées avec des films recommandés "art et essai» par rapport au total des séances offertes en appréciant le nombre de films proposés, la politique d'animation, l'environnement sociologique et cinématographique... Outre ces salles, il existe le réseau des cinéclubs, regroupement de passionnés et possédant une sphère restreinte d'influence (étudiante pour la plupart); celui des salles municipales (appuyées sur les collectivités locales principalement en milieu rural) ainsi que celui des cinémathèques, visant la conservation, la diffusion et la formation aux films de cinéma. Ces dernières ont une fonction de lieu de dépôts de films et à ce titre d'organisation professionnelle poursuivant une mission de service public (la conservation d'un patrimoine culturel) et de diffusion et donc à ce titre d'association d'amateurs revendiquant un cinéma non commercial.

Le phénomène de développement des multiplex a entraîné la protestation des mouvements d'éducation populaire intervenant dans le secteur : ainsi dans la région Rhône-Alpes, l'effet des multiplex sur la fréquentation pour les salles se fait sentir partout où ils se sont ouverts, leur effet principal étant aussi une transformation des formes de la concurrence avec une réorientation de la programmation des salles vers «l'art et l'essai», les films en version originale et le jeune public, entraînant une forme de concurrence en cascade (les salles grand public se rabattant sur l'art et l'essai, les salles d'art et d'essai sur le cinéma de recherche...). Comme le mettent en évidence Léo Anselme et Max Sanier (2001: 3), la «proximité vraiment directe d'un multiplex (moins de 15 minutes) entraîne les effets les plus forts (sur la fréquentation et sur les stratégies associées)", et notamment sur la possibilité pour un même exploitant de proposer en mobilisant ses différentes salles une offre relativement complémentaire et diversifiée à destination du grand public. D'où l'existence selon ces auteurs d'un dilemme fondamental pour ces salles face à l'implantation des multiplex : «Pour les salles soutenues par les collectivités : la modification sociologique des publics; Pour les salles "privées" : la 
disparition pure et simple, si elles ne passent pas dans le giron public ou ne mutent pas en multiplex». Dès lors face à ce développement des multiplex, les positions défendues par les organisations professionnelles sur les multiplex représentent une continuation de la mobilisation sectorielle pour la défense de l'exception culturelle, les discours dominants se situant à trois niveaux : «la défense du cinéma français (et au-delà européen) face au cinéma américain; le soutien d'une création dite culturelle devant le seul objectif commercial de la programmation grand public; et la défense des petits exploitants et cinémas indépendants face aux grands groupements intégrés" (Hayes, $2003:$ : 7). Comme l'illustrent le tableau et l'encadré ci-après, la prise de conscience de ce risque de la part de l'ensemble des cinémas d'Art et essai, aussi bien par l'AFCAE (Association française des cinémas d'art et d'essai) que par le GNCR ou pour les réalisateurs avec I'ACID (Agence du cinéma indépendant pour sa diffusion), s'illustre aussi par le soutien accordé chaque année à une quarantaine de films dans plus de 300 salles à travers la France, accompagnement assuré par la mise en place d'animations diverses : avant premières, reprise ou création de cycles, rencontres avec des réalisateurs, des comédiens, des professionnels du cinéma.

Au plan local des associations regroupant les salles de cinéma indépendantes sont créées, regroupées au plan national au sein du GNCR. Ces associations ont des objectifs ambivalents, provenant de leur double origine (mobilisation de salles associatives, mais aussi relais en partie des politiques publiques du cinéma) : en effet si elles gèrent des activités de service public ("Lycéens au cinéma», "Cinéville») développées pour l'accès de tous à la culture, théoriquement ouverts à l'ensemble des salles, et représentant une part importante de leur budget, elles ont pour objectif de développer l'activité de leurs membres ainsi que de promouvoir certains films (ce qui peut d'ailleurs entrer en contradiction avec l'objectif précédent). L'activité de ces associations vis-à-vis de leurs adhérents se concrétise à plusieurs niveaux. Elles agissent ainsi sur la négociation de films : l'exploitation d'un film se fait sur contrat, avec les structures chargées de la distribution et de la production, au terme duquel un minimum garanti puis un pourcentage de recettes (environ $50 \%$ ) sont demandés, ces exigences pouvant augmenter du fait d'un rapport de force défavorable aux diffuseurs. La concentration du marché de l'exploitation complique l'accès aux films pour les salles de proximité. En effet les distributeurs préfèrent donner leurs films à des salles 
ayant une forte fréquentation. Les multiplex, avec leur poids économique sont alors souvent prioritaires. L'effet de la concurrence n'étant donc pas seulement dans la captation du public mais également dans l'accès aux films, l'association permet une négociation de ces éléments à un point de vue régional (recettes, dates et nombre de copies) en mobilisant le réseau des salles adhérentes.

Autre fonction assurée par ce type d'association, les activités d'animation des salles, travail important sur l'identité des cinémas associatifs et indépendants, consistent en la mise en place d'animations collectives (cycles thématiques, accueil de réalisateurs et comédiens, travail en collaboration avec d'autres associations sur des thèmes de société...). Si le développement dès multiplex constitue une réelle menace pour la survie des cinémas commerciaux grand public des petites et moyennes villes, il semble que les effets soient en partie différents pour les cinémas art-et-essai. Les données tendent à suggérer que l'arrivée d'un multiplex serve à fidéliser les spectateurs de ces salles, surtout là où le cinéma joue un rôle de proximité et de suivi - tarifs préférentiels, actions d'animation en direction des milieux scolaires, séances «jeune public», débats avec réalisateurs. Les cinémas qui s'en tirent le mieux sont précisément ceux qui ont su renforcer leur identité (Delon, 2000, p. 22).

Dans ce cadre, ces cinémas peuvent ainsi mettre en avant leur rapport particulier aux usagers, c'est-à-dire comme une forme d'appropriation par les spectateurs du cinéma dans un cadre d'éducation populaire (cinés clubs, salles associatives permanentes) ou de gestion collective du patrimoine cinématographique par les professionnels (exemple des cinémathèques). Graeme Hayes (2003) va plus loin en inscrivant ce mouvement du cinéma indépendant dans le cadre de mobilisation altermondialiste. Comme le remarque également Armand Mattelart (2002 : 10-11),

«En 2002, lors du deuxième Forum social mondial de Porto Alegre (Brésil), ces mouvements ont placé la diversité culturelle au centre de l'interrogation sur la forme de société possible dans un autre monde possible. Loin de se recroqueviller sur une conception réduite aux seuls produits des industries culturelles, l'exception commerciale culturelle s'est muée en principe de la critique de l'ensemble d'un mode de vie». 
La diversité culturelle a rejoint les débats portant sur les «biens publics communs" que sont la culture, mais aussi l'éducation, la santé et l'environnement, autrement dit tous ces domaines où la séparation des activités humaines entre une sphère économique, sociale ou politique pose question 25 .

Ainsi on peut voir les activités de ces organisations, comme ayant un caractère "socio-technique" marqué, prenant en compte une "multifonctionnalité de la culture», déclinée entre dimension sociale (expression, animation, éducation) et dimension productive (création, loisirs), démarquant fortement les cinémas associatifs du circuit lucratif par leur activité d'accompagnement des films et d'éducation à l'image (Rousselière 2004 a et b) ${ }^{26}$. Cette fonction de déchiffrement / décryptage ou comme l'écrit Jean-Luc Godard (1985) d'interrogation politique sur les images et les sons apparait aujourd'hui indispensable à l'heure même où le spectateur, pour reprendre l'analogie utilisée par Thierry Jousse (2003 : 232) à propos de l'Antre de la folie de John Carpenter, est

"enserré dans les images d'horreur qui figurent plutôt l'horreur de l'image, son aliénation profonde et sa séduction pure, une horreur qu'on peut aisément rapporter au système de reproduction des images à l'infini - stéréotypes et autres trompe-l'œil - organisé et produit par Hollywood, où figurent en bonne place le cinéma et tout aussi bien la vidéo, la télévision, les jeux divers et autres produits dérivés".

\section{Conclusion}

Si ces groupes bénéficient de l'appui de leurs organisations fédératives (promotion collective, formation, aide à l'accès au film, accompagnement...) dans un contexte de concentration de l'amont (accès au film) et de l'aval (diffusion du film) ou de la diversification par la technique des sources potentielles de profit (DVD), si cela peut même aller en de rares exemples jusqu'à aboutir à une filière alternative (comme le montre le succès du film la Prophétie des Grenouilles produit par une filiale d'une association d'éducation populaire et qui a pu être distribué et exposé en s'appuyant sur les écrans des réseaux associatifs), l'avenir semble toutefois rester celui de la négociation de marges de manœuvre au sein d'un univers industriel fortement contraignant.

Dans ce cadre, on peut alors s'interroger sur l'impact de la 
transformation des politiques publiques, jouant un rôle important en France et dans d'autres pays européens (Vivancos, 2000) et devant s'adapter à de nouveaux objectifs affichés par les Pouvoirs Publics qui ne sont plus ceux de «l'exception culturelle» mais de «la diversité culturelle", et ceci alors même que de nombreuses critiques des politiques publiques d'aide au cinéma se font jour comme avec Jean Cluzel (2003). La situation du cinéma en France, régulé par un dispositif politique, administratif, financier et symbolique mis en place depuis trois quarts de siècle, s'incarnant notamment dans la création en 1946 d'un outil de politique nationale comme le CNC (Centre national de la cinématographie), témoigne d'une transformation historique des modalités d'intervention de l'État dans l'économie du cinéma (Farchy, 1999 : 190-193) par le passage d'une politique industrielle de structuration du secteur dans l'après guerre à une politique à prétention culturelle avec le rattachement du cinéma au Ministère de la Culture en 1959. Alors qu'elles doivent rentrer dans le cadre d'une négociation prochaine en 2005 au sein de l'Union européenne, ce sont ces formes de l'aide qui apparaissent en crise actuellement puisqu'en mettant l'accent sur l'aide à la production plutôt que sur l'aide à la diffusion des films (comme avec l'aide aux salles) elles accentuent le décalage entre le niveau élevé de la production et les possibilités limitées de sortie des films dans de bonnes conditions ${ }^{27}$.

Comme le rappelle Claude Forest (1998), la diversité artistique et culturelle des œuvres de cinéma s'accompagne donc et se nourrit d'une diversité de la population des opérateurs économiques du secteur. Alors que les enjeux culturels sont au cœur de la constitution de l'Europe comme une réalité politique non réductible à une vaste zone de libre échange, si l'Europe veut donc continuer à produire un cinéma qui lui serait spécifique, développer "son génie (...) dans le dialogue des pluralités qui produit le changement» (Morin, 1990 : 149 ), elle ne peut faire l'économie d'une réflexion forte sur les conditions de l'exposition de sa production et préserver une pluralité de réseaux d'exploitation (Thiollière, Ralite, 2003).

\author{
Damien Rousselière \\ Allocataire-moniteur en économie \\ à l'université Pierre Mendès France \\ (Grenoble II)et Membre du LEPII \\ (Laboratoire d'économie de la production \\ et de l'intégration internationale)
}




\section{Damien Rousselière}

\section{Bibliographie}

ANSELME L., SANIER M. (2001) "Les salles traditionnelles face aux multiplex en RhôneAlpes", Rapport de l'ARSEC pour le CNC et la Région Rhône-Alpes, septembre.

ARIEs P. (1997), Les fils de Mc Do, La macdonalisation du monde, Paris, L'Harmattan.

BAER J.M. (2003), L'exception culturelle- une règle en quête de contenus, Paris, En Temps réel.

Ballynlich A. (2000) "Vivendi-Universal : la clé, c'est le portail», Cahiers du Cinéma, $n^{\circ} 552$, décembre.

BENGHOZI P.-J. (2003), "De l'exception à la diversité : quel avenir pour le pluralisme culturel?» in Benghozi P.-J., Paris T., Saint-Pulgent M. de, Mondialisation et diversité culturelle. Le cas de la France, Paris, IFRI, Les Notes de l'Ifri $n^{\circ} 51$ - Réactions et réponses à la mondialisation, p. 55-64.

Benhamou F. (2003) L'économie de la culture, Paris, La Découverte, Repères.

Boltanski L., ThÉvenOt L. (2000) “The Reality of Moral Expectations, A Sociology of Situated Judgement", Philosophical Explorations, vol. 3, n ${ }^{\circ} 3$, p. 208-231.

BONNELL R. (2001) La 25eme image, Paris, Gallimard.

BOURDIEU P. (1994) Raisons pratiques, Paris, Le Seuil Points.

Bourdieu P.(1996) Sur la télévision, Paris, Liber, Raisons d'Agir.

Bourdieu P. (1998) Les règles de l'art, Paris, Le Seuil Points.

Chabrol C. (2003), Comment faire un film, Paris, Manuels Payot. de France.

Cluzel J. (2003) Propos impertinents sur le cinéma, Paris, Presses Universitaires

Creton L. (dir.) (1998) Cinéma et (in)dépendance, Une économie politique, Paris, Presses de la Sorbonne Nouvelle, Théorème $n^{\circ} 5$. Cinéma.

Creton L. (2001) Économie du cinéma, Perspectives stratégiques, Paris, Nathan

Delon F. (2000) Les multiplex, Rapport au Ministre de la culture et de la communication, La Documentation Française.

Demoustier D., Rousselière D., Clerc J.-M., Cassier B. (2003) “L'entreprise collective : unité et diversité de l'économie sociale et solidaire", Revue internationale de l'économie sociale, vol. 82, n² 290, p. 56-73.

Demoustier D., Rousseliere D. (2005) "Social Economy as Social Science and Practice : Historical Perspectives on France" in Clary J., Dolfsma W., FigarT D. (dir.), Ethics and the Market : Insights from Social Economics, London \& New York, Routledge, Advances in Social Economics. (Forthcoming).

FARCHY J. (1999) La fin de l'exception culturelle, Paris, CNRS Éditions.

FOREST C. (1998) "Le jour de la dépendance, Exportation cinématographique américaine et positionnement des intervenants français de la filière", in CRETON L. (dir.) p. 123-139.

FOREST C. (2002) L'argent du cinéma, Paris, Belin éditions, Économie.

Godard J.-L. (1985) Godard par Godard, Des années Mao aux années 80, Paris, Flammarion. 
Hansmann H. (1996) The Ownership of Enterprise, Cambridge, The Belknap Presss of Harvard University Press.

HAYES G. (2003) «Mobilisation anti-multiplex : où l'action locale retrouve l'exception culturelle", Communication au colloque "les mobilisations altermondialistes», Paris, 3-5 décembre.

Jousse T. (2003) Pendant les travaux, le cinéma reste ouvert, Paris, Éditions Cahiers du Cinéma, Essais.

MalrauX André (1946) Esquisse d'une psychologie du cinéma, Paris, Nouveau Monde Éditions, 2003.

Marx K. (1857) Introduction à une critique de l'économie politique in CEuvres, Philosophie, Tome III, Paris, Gallimard, La Pléiade, 1990.

MATTELART A. (2002) "La diversité culturelle : entre histoire et géopolitique", Actes du colloque 2001 Bogues, Globalisme et Pluralisme, Montréal, 24-27 avril.

Mattelart A., Neveu E. (dir.) (1996), Dossier "Cultural Studies», Réseaux, n 80.

Mattelard A., Neveu E (2003) Introduction aux Cultural Studies, Paris, La Découverte.

Morin E. (1956) Le cinéma ou l'homme imaginaire, Paris, Éditions de Minuit.

Morin E. (1990) Penser /'Europe, Paris, Gallimard, Folio Actuel.

Paris T. (2004) «Diversité culturelle et Mondialisation" in MontBriaL DE T., MoreaU Defarges P. (dir.) RAMSES 2005, Les faces cachées de la mondialisation, Paris, Dunod.

Prado C. (2003) «Entre succès fragiles et loi de l'argent, Le cinéma indépendant marginalisé», Le Monde Diplomatique, mai, p. 27.

OEA (Observatoire européen de l'audiovisuel) (2003) Tendances du marché mondial du film, Focus.

REICH R. (1993) The Work of Nations, New York, Alfred A. Knopf Inc. p. 845-858.

Rosen S. (1981) “The Economics of Superstars», American Economic Review, vol. 71,

Rousselière D. (2004 a), «L'économie sociale dans le secteur culturel» in Girard D. (dir.), Solidarités collectives, Tome l, Paris, L'Harmattan.

RousSELIĖE D. (2004 b) "Concentration de la diffusion du cinéma et diversité culturelle", Post-Scriptum, Revue de recherche interdisciplinaire en textes et médias, $n^{\circ}$ spécial : "L'Europe au Cinéma», vol. $3, n^{\circ} 4$.

Scort A. (2000) The Cultural Economy of Cities, London, SAGE Publications, Theory, culture and Society.

SIROËN J.-M. (2000) «Le cinéma, une industrie ancienne de la nouvelle économie», Revue d'économie industrielle, $n^{\circ}$ 91, p. 93-108.

THIOLLIÈRE M., RALITE J. (2003) Exploitation cinématographique : le spectacle est-il encore dans la salle?, Rapport d'information de la commission des affaires culturelles, Sénat, Rapport $n^{\circ} 308,21$ mai (disponible sur http://www.senat.fr).

Vivancos P. (2000) Cinéma et Europe : réflexions sur les politiques européennes de soutien au cinéma, Paris, L'Harmattan.

WAYNE M. (2003) «Post-Fordism, monopoly capitalism, and Hollywood's media industrial complex», vol. 8, $n^{\circ} 1 ;$ p. 82-103. 
1. La concentration des producteurs et des produits, se traduisant non par le nombre de films (stable ou en augmentation) mais par le coût moyen de production et la concentration du nombre de spectateurs; la mise en question des modes de financements de la création (l'économie de l'avance sur recettes en France par exemple) cf. (Créton, 2001).

2. Allant de $11 \%$ en Italie à $48 \%$ en Belgique.

3. Voir à ce propos différents articles dans les Cahiers du Cinéma en 2001 et 2002 par Élisabeth Lequeret.

4. Ce dont Karl Marx lui-même se défiait, comme en témoigne son éloge de l'art grec et de Shakespeare comme modèles artistiques «inaccessibles» (Marx, 1857).

5. Face à la critique de "scientisme" dès lors que l'on s'intéresse à l'économie des pratiques culturelles, on se permet ici de rappeler ce qu'écrivait Pierre Bourdieu à propos de l'œuvre littéraire : «l'analyse scientifique des conditions sociales de la production et de la réception de l'œuvre d'art, loin de réduire ou de la détruire, intensifie l'expérience littéraire" (Bourdieu, 1998 : 14-15).

6. Pour une discussion plus précise de la notion de diversité culturelle (et de son historique), se référer aux travaux de Armand Mattelart (2002), critiquant «la diversité culturelle assimilée [uniquement] à la multiplication de l'offre et de demande sur le marché des biens culturels". Différents termes sont ainsi en confrontation (Paris, 2004). Ainsi l'exception culturelle est apparue dans le cadre des négociations de I'OMC (Organisation mondiale du commerce) en 1993 et signifiait le refus de l'Europe et de la plupart des Etats membres de l'OMC de prendre des engagements de libéralisation du secteur audiovisuel, et leur volonté de préserver leur capacité à intervenir dans ce secteur, notamment par des instruments de politique audiovisuelle. La notion de diversité culturelle est apparue dans les années 1990 : l'article 1 de la déclaration universelle de l'UNESCO sur la diversité culturelle le 2 novembre 2001 dont l'article 1 stipule que ula culture prend des formes diverses à travers le temps et l'espace. Cette diversité s'incarne dans l'originalité et la pluralité des identités qui caractérisent les groupes et les sociétés composant l'humanité. Source d'échanges, d'innovation et de créativité, la diversité culturelle est, pour le genre humain, aussi nécessaire qu'est la biodiversité dans l'ordre du vivant. En ce sens, elle constitue le patrimoine commun de l'humanité et elle doit être reconnue et affirmée au bénéfice des générations présentes et des générations futures". Le pluralisme culturel "constitue la réponse politique au fait de la diversité culturelle» (déclaration de l'UNESCO) : si la diversité est un état de nature, le pluralisme apparaît comme la volonté politique d'assurer la cohabitation des diverses cultures. La cohabitation culturelle pour Dominique Wolton répond au défi politique qui va au-delà de la seule question culturelle, "de respecter les identités culturelles, tout en évitant le repli sur soi, et sa dérive ethniciste ou communautariste». Voir également pour une histoire de la notion de diversité culturelle (Baer, 2003).

7. Notons que l'on arrive à la même conclusion si on étudie l'évolution de la marge bénéficiaire de ces différents acteurs (Thiollière, Ralite, 2003).

8. Cette progression rencontre actuellement des limites réglementaires dans la mesure où la salle ne peut recueillir moins de $50 \%$ de la recette hors taxe.

9. En effet, au sein d'un secteur où les différentes branches sont traditionnellement rémunérées au pourcentage de la recette par entrée, l'introduction de telles formules faisait difficulté : elle ne permettait plus d'individualiser, pour chaque film, les recettes issues des abonnements et rendait par conséquent problématique la rémunération des distributeurs et des ayants droit. 


\section{Damien Rousselière}

10. Voir à ce titre les différentes données disponibles sur le site du CNC : www.cnc.fr

11. En droit français (mais cela rejoint en grande partie la définition européenne), il s'agit des complexes cinématographiques d'au moins 8 salles pour une capacité minimum de 1000 fauteuils.

12. Par exemple le 2 janvier 2004, quatre films (Le monde de Némo, le Seigneur des Anneaux, Scary Movie, les Ripoux 3) occupaient 3022 écrans (sur 5280 ) soit $57 \%$ de l'ensemble des écrans (tous réseaux confondus) !

13. L'ensemble des programmateurs appelant l'ensemble des distributeurs le lundi matin, au vu des résultats dont tous ont eu communication en fin de week-end.

14. L'amplitude du choix potentiel incite certains à toucher tous les segments de la clientèle, y compris la plus cinéphilique, livrant une concurrence frontale avec la frange des exploitants "art et essai» qui s'étaient établis sur cette niche, ne seraitce que parce que la programmation de films "art et essai» porteurs (l'exemple type pour le cas français étant le film annuel de Woody Allen) permet de diversifier les sources de profit en assurant une durée d'exploitation optimale de chaque film.

15. On ne compte plus les accords de partenariat entre les sociétés de multiplex et celles de restauration rapide à proximité pour proposer des offres couplées préférentielles.

16. Dans un article des Cahiers du Cinéma, Anne Ballynlich (2000) avait caractérisé de cette manière la stratégie des grandes entreprises des industries culturelles.

17. De même que le système français finance largement des cinéastes étrangers, conduisant par exemple à ce que Denys Arcand puisse recevoir pour les Invasions Barbares le césar du meilleur film. On peut y voir alors une nouvelle forme de rapport centre-périphérie (France par rapport au reste du monde francophone).

18. Un simple coup d'œil sur les affiches (se réduisant à une collection de portraits des acteurs) des principaux "blockbusters" français montrent qu'ils singent jusqu'à la caricature l'image et l'univers des films américains.

19. Comme Arte, elles tendent de développer ainsi un compromis sur la diffusion du film entre un produit télévisuel et un film indépendant plus "classique» (par exemple en proposant de manière légèrement décalée une première vision télévisuelle puis une sortie en salle).

20. L'analyse spécifique de la situation du documentaire et de la part de plus en plus importante que les chaînes occupent dans "son formatage télévisuel» nécessiterait à elle seule un développement beaucoup plus large que les quelques arguments mentionnés ici relativement à l'économie générale du film.

21. Rappelons ici que selon les chiffres du CNC pour 2002, $5 \%$ des titres représentent $73 \%$ des ventes de DVD; les films américains représentent une part identique des ventes.

22. Pour ce dernier cas, l'effet est même celui de la contamination sur la structure du récit du film se résumant à celle d'un jeu vidéo (action, récit préalable à l'action suivante, action suivante de difficulté supérieure, etc.)

23. Claude Forest y voit un avantage comparatif de Hollywood : "la différence incontournable entre les régimes juridiques anglo-saxons et ceux d'autres pays comme la France fonde l'abîme entre les différentes conceptions de l'auteur de l'œuvre à produire comme de l'œuvre elle-même. Les États-Unis n'ont, en effet, pas adhéré à la convention de Berne sur les droits d'auteur qui donnent à ceux-ci 
(que ce soit pour un livre, un scénario ou toute autre œuvre d'art) un droit de regard absolu sur l'utilisation ultérieure qui sera faite de leur création. II s'ensuit que, en droit américain, seul compte le propriétaire du copyright, qui est cessible contractuellement entre n'importe quelles parties, comme par exemple un scénariste salarié et son employeur" (Forest, $2002: 219$ ).

24. Cette analyse canonique (la "politique des auteurs" des Cahiers du Cinéma) nécessiterait toutefois un réexamen. En témoigne le destin du film «Gangs of New York", ayant largement échappé (dans son montage, dans les scènes coupées...) au contrôle de Martin Scorsese, pourtant considéré par la Revue comme l'archétype du«contrebandier du cinéma américain».

25. On se permet de renvoyer ici à une revue de la littérature sur les approches théoriques de l'économie sociale comme organisation (ensemble des associations coopératives mutuelles i.e. des organisations productives aux règles de fonctionnement particulières) ou comme champ d'activité (où il est difficile de séparer au plan théorique, de différencier une dimension économique - qui pourrait être celle du calcul rationnel - d'autres dimensions - faisant intervenir d'autres principes de légitimité / justification) (Demoustier, Rousselière, 2005).

26. Comme le résume bien une des structures associatives rencontrées, "que ce soit à travers le cinéma, les spectacles vivants, les expositions, les activités d'expressions culturelles, toutes les actions qui nous développons tendent vers une même exigence : ouvrir et nourrir les esprits, mieux se connaître et mieux se comprendre". Le cinéma dans ce type d'organisation s'inscrit avant tout dans un projet social et éducatif plus vaste.

27. Pour une part, certaines politiques réglementaires ont été mises en place. Outre l'encadrement des cartes illimitées, des engagements de programmation pris par les opérateurs doivent contribuer à la diversité de la programmation des multiplex en prévoyant un pourcentage minimum de séances consacrées aux films européens et en limitant la pratique de la multidiffusion consistant à projeter le même film dans plusieurs salles d'un multiplex. 